\title{
Measuring Domain Shift for Deep Learning in Histopathology
}

Karin Stacke, Gabriel Eilertsen, Jonas Unger and Claes Lundström

The self-archived postprint version of this journal article is available at Linköping University Institutional Repository (DiVA):

http://urn.kb.se/resolve?urn=urn:nbn:se:liu:diva-170816

N.B.: When citing this work, cite the original publication.

Stacke, K., Eilertsen, G., Unger, J., Lundström, C., (2020), Measuring Domain Shift for Deep Learning in Histopathology, IEEE journal of biomedical and health informatics.

https://doi.org/10.1109/JBHI.2020.3032060

Original publication available at:

https://doi.org/10.1109/JBHI.2020.3032060

Copyright: Institute of Electrical and Electronics Engineers

http://www.ieee.org/index.html 


\title{
Measuring Domain Shift for Deep Learning in Histopathology
}

\author{
Karin Stacke, Gabriel Eilertsen, Jonas Unger, and Claes Lundström
}

\begin{abstract}
The high capacity of neural networks allows fitting models to data with high precision, but makes generalization to unseen data a challenge. If a domain shift exists, i.e. differences in image statistics between training and test data, care needs to be taken to ensure reliable deployment in real-world scenarios. In digital pathology, domain shift can be manifested in differences between whole-slide images, introduced by for example differences in acquisition pipeline - between medical centers or over time. In order to harness the great potential presented by deep learning in histopathology, and ensure consistent model behavior, we need a deeper understanding of domain shift and its consequences, such that a model's predictions on new data can be trusted.

This work focuses on the internal representation learned by trained convolutional neural networks, and shows how this can be used to formulate a novel measure - the representation shift - for quantifying the magnitude of model-specific domain shift. We perform a study on domain shift in tumor classification of hematoxylin and eosin stained images, by considering different datasets, models, and techniques for preparing data in order to reduce the domain shift. The results show how the proposed measure has a high correlation with drop in performance when testing a model across a large number of different types of domain shifts, and how it improves on existing techniques for measuring data shift and uncertainty. The proposed measure can reveal how sensitive a model is to domain variations, and can be used to detect new data that a model will have problems generalizing to. We see techniques for measuring, understanding and overcoming the domain shift as a crucial step towards reliable use of deep learning in the future clinical pathology applications.
\end{abstract}

\section{INTRODUCTION}

A fundamental part of machine learning is the problem of generalization, that is, how to make sure that a trained model performs well on unseen data. The problem is particularly prevalent for deep learning algorithms where the tremendous capacity of a deep neural network (DNN) can easily overfit to the exact features of the training data, resulting in poor generalization. If the unseen data has different distribution, i.e. a domain shift exists, the problem is significantly more difficult $[1,2]$ - even the smallest changes in the statistics as compared to the training data can cause a DNN to fail completely [3]. Domain shift, defined as differences between the source and target domain data, can be caused by, for example, differences due to sampling bias, differences in image content

K. Stacke is with the Department of Science and Technology at Linköping University, Sweden, and Sectra AB, Sweden, e-mail: karin.stacke@liu.se.

G. Eilertsen and J. Unger are at the Department of Science and Technology at Linköping University, Sweden, and at the Center for Medical Image Science and Visualization, Linköping University, Sweden.

C. Lundström is at the Center for Medical Image Science and Visualization, Linköping University, Sweden and Sectra AB, Sweden.

Manuscript received XX

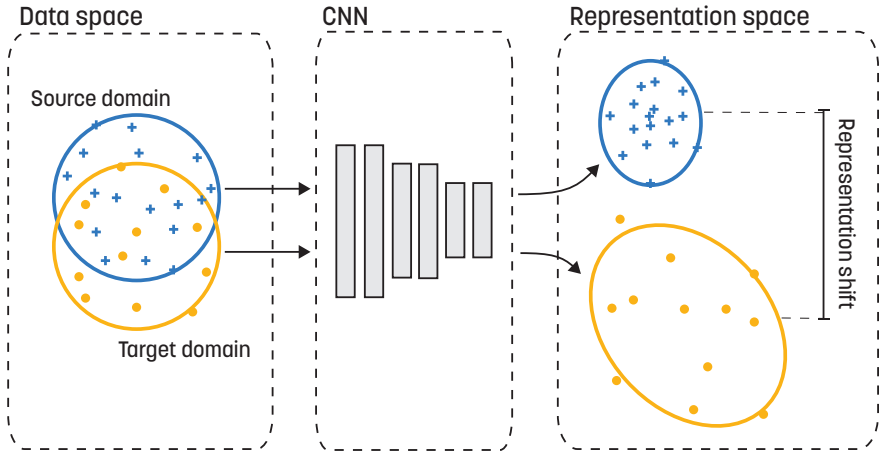

Fig. 1: Illustration of the model dependent nature of domain shift between two datasets. The sensitivity of a neural network can make the representations of source and target domains diverge, and the domain shift has to be measured in the representation space.

or view angle, or differences in image characteristics such as brightness, noise or color [4]. How well a model can generalize from a source domain to a target domain depends on the form of domain dissimilarity.

In the case of digital pathology, domain shift challenges in whole-slide-imaging (WSI) are typically ascribed to color, brightness and contrast variations, caused by stain variations and scanner properties [5]. The definition, however, also includes differences introduced by different tissue and/or staining type. Training robust models which are invariant to some domain shift changes (such as color variations which may occur over time) is crucial for patient safety, and detecting when a domain shift exists is an important initial step to ensure model performance.

In this paper, we take a closer look at the representations learned by a convolutional neural network (CNN) trained on histopathology data, and present a way to quantify the differences in how datasets are represented. Our experiments, done using two datasets and two model architectures show that a tumor classifier learn very different feature representations depending on how the training data is pre-processed. We also present a novel metric, the representation shift, to measure the statistical difference between source and target domains, and demonstrate a correlation between the representation shift and the drop in classification accuracy on images from a new domain. Together, the experiments help us shape a better understanding on what a tumor classifier has learned and how sensitive its representation is to changes in data distribution. The presented metric for measuring the domain shift in the 
learnt representation space gives valuable insights for detecting data which the model has not seen during trained. We believe that this understanding is crucial for future development of reliable deep models that are robust to domain variations and thus possible to use in a real-world clinical pipeline.

The main contributions can be summarized as follows:

1) We use the filter activations of a trained model to define a novel representation shift; an unsupervised formulation of a measure that captures differences in feature representation caused by test data from a different distribution than the training data.

2) We analyze the test accuracy of CNNs trained for tumor classification on hematoxylin and eosin (H\&E) stained images: how it changes with different models, test data and augmentation strategies, and how it relates to the presented metric.

3) We compare the measured domain shift to two previous methods in domain difference analysis and uncertainty estimation, on two different histopathology datasets, and demonstrate a better correlation between measured shift and the final drop in accuracy.

A preceding version of this paper has been presented at the MICCAI 2019 workshop on Computational Pathology (COMPAY) [6]. Building on this work, we make a number of larger extensions, including experiments on a second dataset and comparison to two previous methods. We compare to two additional discrepancy metrics for calculating the representation shift, and analyze the differences in measuring the representation shift on different layers. We also provide a deeper investigation on how the sensitivity of neural networks affects the learned representation, using techniques for feature visualization and dimensionality reduction.

\section{BACKGROUND}

In this section, we first discuss the concept of domain shift and then summarize related previous work, with a particular focus on the histopathology area.

\section{A. Domain shift}

One of the central problems in machine learning is that of generalization. A model trained on a certain type - or domain - of data, cannot be expected to generalize well to a completely different domain. For example, generalizing from hand drawings to camera captured images is not expected to work well. However, as some data variability is expected, it is of importance to handle this variance, or at least know when it is likely to impact performance significantly. This shift in domain statistics is especially pronounced for deep learning algorithms, where over-parameterized and high capacity models can easily become locked to fine details of the training set. Thus, the domain shift problem is closely linked to the particular model and optimization scheme, as illustrated in Figure 1.

The convolutional layers of a convolutional neural network (CNN) maps an input image to a representation, which for each layer becomes increasingly more abstract. The fully connected layers relies on this representation to, for example, infer class labels in a classification task. This can be thought of as drawing decision boundaries between the different classes within the representation space. Thus, the representation has to capture the information required to solve the task at hand. However, there are no mechanisms ensuring that the representation does not depend on features that are not explicitly required, such as domain specific details in color statistics. With the capacity of neural networks, this means that the representation can be highly sensitive to small changes in image statistics. The sensitivity problem has been under intense investigation, where methods for defining adversarial examples have demonstrated how even perceptually unnoticeable perturbations in an image can force a model to predict any output class with high confidence [7, 8]. Such perturbations can also be created without access to a trained model [3], and by performing natural image operations $[9,10,11]$. Altogether, this means that although the differences between the two domains could be perceptually small in the image space, the dependence on training data statistics can force the domains apart when considering differences in internal model representation, as illustrated in Figure 1.

\section{B. Related Work}

How to mitigate the domain shift problem has been a topic of extensive study, for machine learning in general and deep learning in particular. Two different families of approaches can be recognized: domain generalization and domain adaptation. Both these approaches assume that the model's target is the same for both source and target domain. Domain generalization does not use any target domain data, while this is required in domain adaptation.

Domain adaptation: Many methods have been proposed for transforming data and models across domain shifts, both in supervised and unsupervised settings. In deep learning, a common objective is to align the representation of different domains within a model. This can be done by explicitly comparing and minimizing the discrepancy between representations $[12,13,14]$, or by having an adversary that tries to discriminate between source and target domains [15, 16, 17]. For a thorough explanation of previous work on domain adaptation we refer to recent surveys on the topic $[18,4]$.

A few domain adaptation methods have also been presented for histopathology. Lafarge et al. [19] use a domain adversarial network, that during training tries to remove domain-specific knowledge. However, they saw that the best results still were in combination with color augmentation. Furthermore, some presented methods utilize generative adversarial networks (GANs) [8] for domain adaptation or stain normalization. These consider a whole domain of target images instead of only depending on one reference image as in previous stain normalization methods. For example, Zanjani et al. use adversarial auto-encoders for this purpose [20]. Ren et al. [21] use adversarial training together with a Siamese network for domain adaptation, while Shaban et al. [22] and de Bel et al. [23] rely on Cycle-GANs [24]. Xing et al. [25] also use cycle-consistent adversarial training but for domain adaptation across modalities. 
Domain generalization: Previous work in domain generalization often try to learn unified representations from multiple source domains [26, 27, 28]. The source domains can also be created artificially from a single source domain, e.g. by means of GANs [29]. Another technique, termed domain randomization, aim at accomplishing domain generalization by forcing a deeper understanding of images by creating nonrealistic variations of image compositions [30, 31].

The problem of domain generalization in pathological images from different medical centers and different scanners has been discussed in previous work. Tellez et al. [32] show that augmentation of the data increase generalizability to new datasets, where color stain augmentation was the technique which by itself had the most positive affect. Vahande et al. [33] and BenTaieb et al. [34] present two different ways of doing stain normalization (the latter a learning based method similar to [22]), and argue the importance of normalization rather than color augmentation. Arvidsson et al. [35] and Ciompi et al. [36] both noted the importance of either color augmentation or stain normalization for good generalization performance. Tellez et al. [37] investigate how much improvement color augmentation and/or stain normalization have on generalization performance to new datasets. Their results showed that a combination of the two gave the best results, and concluded that color stain augmentation always should be used.

Neural network understanding: Much research has been devoted to understand DNNs trained with natural images, for example what representations the model learns, how the choice of loss function and batch size affects the representation [38, 39, 40] and how this can be leveraged for transfer learning, novelty detection and detection of adversarial examples [39, 41, 42]. However, the relevance of these research results for histopathology is unclear, as the data characteristics substantially differ from natural images. For medical imaging examples in radiology and ophtalmology, Raghu et al. [43] observed distinct differences in the models learned compared to ImageNet training.

Similar to previous work in domain adaptation and generalization, we look at the shift between different image domains. However, we are interested in studying its properties and how it can be measured reliably in histopathology applications. We do this in a statistical comparison between distributions of the representations within a neural network. Thus, the focus is on finding a suitable deep representation and comparing the statistics of this between datasets from different domains and for different training data transformations. This is a significant difference as compared to previous work in domain adaptation/generalization; while these attempt at alleviating the problem of domain shift, they do not provide viable methods for measuring its significance.

\section{Domain Shift Challenges in Histopathology}

In digital pathology, the domain shift problem is commonly observed between WSIs captured at different medical centers, due to differences in slide preparation, staining protocol, scanner properties, etc. Data from the same medical center can also experience shifts in domain statistics, for example from changes in the capturing pipeline over time. For deep learning algorithms, which has become one of the most promising techniques for medical imaging technology, this means that care needs to be taken when deploying a model as aid in a diagnosis pipeline. As a crucial part of this process, we need an understanding on when a model is going to perform as expected.

In this section, we expand on the motivation for domain shift analysis methods in histopathology imaging. The rationale includes examples demonstrating a high variability of learned representations, and a distinct difference from filter characteristics for natural images.

\section{A. Maximum activations}

As previously discussed, small variations in input data may cause significant differences in the learned representation space. In order to visualize such sensitivity of the representation in the convolutional layers of a $\mathrm{CNN}$, we derive artificial images that maximize the activations of individual filters of the deepest convolutional layers of CNNs. The images are initialized with noise, followed by gradient ascent in order to optimize towards images that maximize filter activations $[44,45]$. This type of feature visualization has been utilized extensively for natural images, and it is interesting to investigate what it may reveal about training models with histopathology images.

Figure 2 shows selected images of these maximally activating feature images, extracted from the final convolutional layer. The optimization has been performed on two different models and with four different setups of the training data. We refer to Section $\mathrm{V}$ for a detailed explanation of the models and training data. The maximum activation optimization is not conditioned to keep images within the range of allowed pixel values, which means that the resulting color palette is unrealistic. Comparing the different training data setups (rows) we see a distinct lack of color in the second rows, indicating that models trained with color augmentation to a larger extent than the other models have learned to ignore the color components in the images.

Images shown in Figure 2b (from Mini-GoogLeNet) reveal that the models have learned cell-like circular structures. Both stain normalization and CycleGAN transformation unifies the color variations in the dataset, which is visible by a more homogeneous color scale in these images. The Simple CNN architecture (2a) seems to find more low-level structures, which might indicate that the model has not learned the expected features which separate tumor cells from non-tumorous tissue.

In summary, the maximum activation features point to the high sensitivity of training setup, where differences in model architecture and training data formulation have a crucial impact on the representation learned by a model.

\section{B. Filter characteristics}

Another aspect motivating domain shift research specific for histopathology is the characteristics of learned filters, which may be different from what we know about natural images. As Raghu et al. observed in [43], models trained on medical 


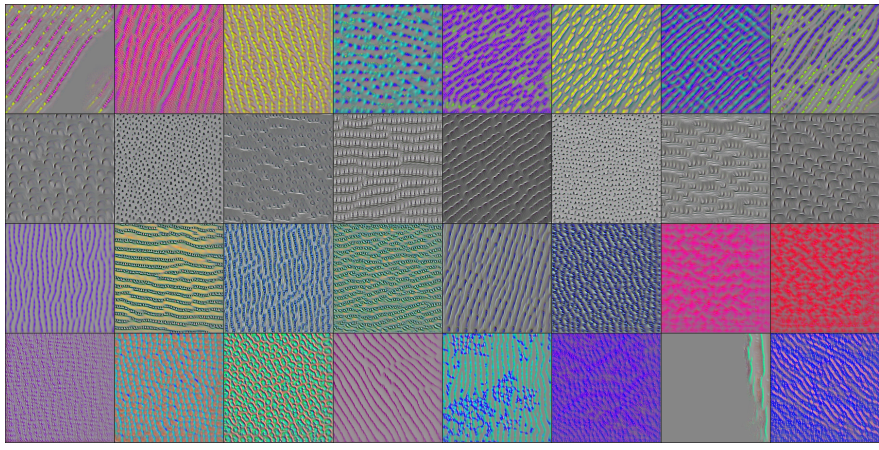

(a) Simple CNN, the last convolutional layer

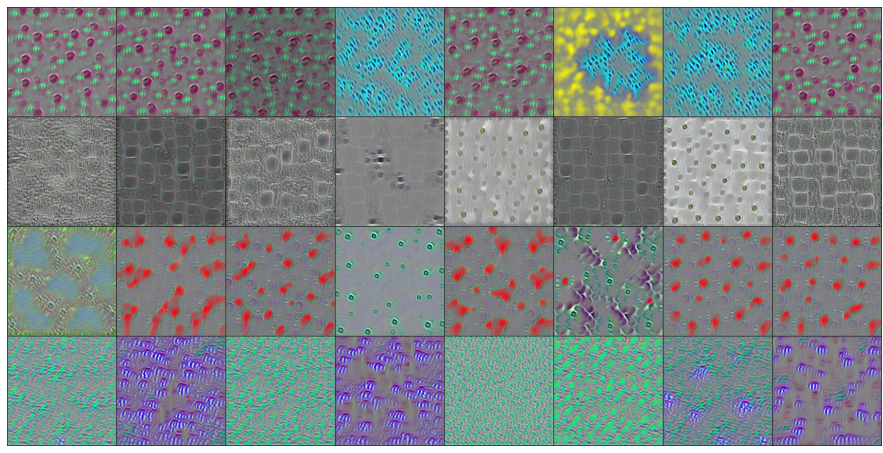

(b) Mini-GoogLeNet, the convolutional layer in the first auxiliary branch

Fig. 2: Each row shows example images that maximally activates different filters in models Simple CNN (a) and MiniGoogLeNet (b), trained with (from top to bottom): original data, color augmentation, stain normalization, and CycleGAN. Best viewed digitally.

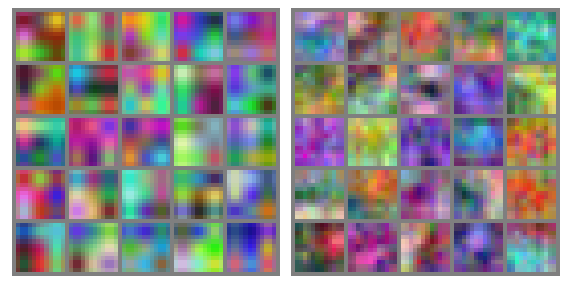

Fig. 3: Visualizations of non-Gabor-like filters from the first convolutional layer for Simple CNN (left) and MiniGoogLeNet (right) architectures, trained with histopathological data. See Section V for model definitions. Best viewed in color.

image datasets did not learn Gabor filters, which were the case when trained on ImageNet [46]. The same is true for models trained with histopathology data, as demonstrated in Figure 3. While Gabor filters are known to be a common denominator between CNNs trained for different tasks and on different data, this counter-example motivates us to look closer at how CNN optimization is shaped by histopathology data, and what the implications are for generalization to unseen domains.

\section{QUANTIFYING DomaIN SHIFT}

Measuring model performance on a new target dataset can easily be done if the target labels are available. However, this is often not the case, as annotation of new data can be both time consuming and error prone, making this method for continuous monitoring of a deployed model unfeasible. A naïve approach of detecting differences in data would be to expect the softmax probabilities of the model output to reflect the model's uncertainty of new, unfamiliar data. However, softmax probabilities are known to be miscalibrated and not represent model uncertainty well $[47,48]$. In order to provide a more reliable measure of uncertainty using softmax probabilities, an ensemble of models can be used [49]. However, as we will see in the comparisons in Section VI, this is also problematic. A better metric for detecting unfamiliar (domain shifted) data is thus needed.

In the domain adaptation literature, a common approach for minimizing the discrepancy between two datasets is to minimize the discrepancy between distributions of activations of hidden layer(s). As the shift between image distributions can linger in the deeper layers, the differences in distributions of activations from deeper layers can be used to model the dataset shift $[14,50]$. In [51], the authors compare the second order statistics of the distributions of the activations from one layer between source and target data, showing that a simple minimization in this discrepancy leads to better generalization to the target data. In this work we are not presenting a new way of domain adaptation, but rather leverage the knowledge from this field to introduce a metric for measuring when domain generalization has succeeded.

Building on the idea of [51], we propose the representation shift metric, which measures the differences in distributions of the learned feature representation, comparing the training set (source) to a dataset from a different domain (target). This way, we can quantify the domain shift, not in image space, but in the model specific latent representation space. In a "well-trained" model, a deeper convolutional layer spans a space which corresponds only to image features which are relevant for the specific task at hand, irrelevant features should have been discarded. Looking at the statistical distributions of activation values for each filter in one of the layers, we can see how the model has described a particular dataset in this latent representation. By comparing this description with the description of a second dataset we can measure statistical discrepancies $\mathcal{D}$ between them. If the model has succeeded in learning only relevant features, the distributions for each filter should be similar (i.e., small distances). If this is not the case, then the representation of the first dataset depends on features not present in the second dataset (or vice versa), likely caused by domain shift in the image domain. There is then an increased risk of a decrease in model performance. (The effective performance will also depend on the final fully connected layers).

Below follows a detailed description of the metric, and we refer to Figure 4 for a schematic overview.

Consider a CNN with layers $\left\{l_{1}, \ldots, l_{L}\right\}$. Let $\Phi_{l}(x)=$ $\left\{\phi_{l 1}(x), \ldots, \phi_{l k}(x)\right\}, \phi_{l k}(x) \in\left\{\mathbb{R}^{h \times w}\right\}$ denote the layer activations at layer $l$ and filter $k$, produced by input sample $x$. The mean value of each $\phi_{l k}(x)$ is denoted $c_{l k}(x)$, given by 


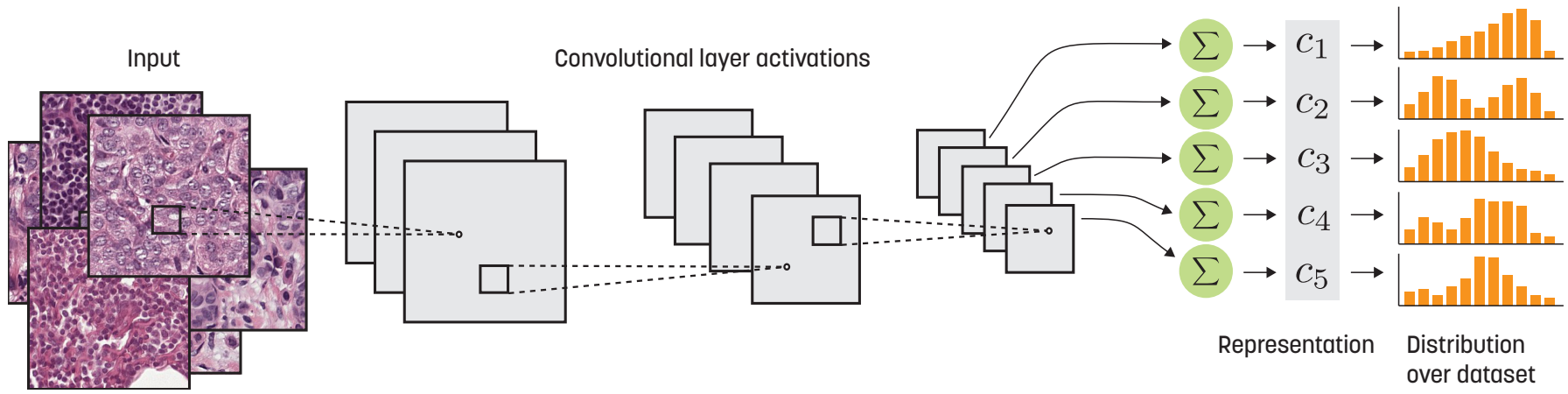

Fig. 4: Schematic view of how CNN representations are estimated. The distributions of each dimension of the representation are compared between datasets in order to measure the representation shift (see Figure 1).

$$
c_{l k}(x)=\frac{1}{h} \frac{1}{w} \sum_{i, j}^{h, w} \phi_{l k}(x)_{i, j},
$$

where $h$ and $w$ are the height and width of the two-dimensional layer activation $\phi_{l k}(x)$. Empirical analysis showed very little variance of $\phi_{l k}(x)$, indicating that the mean value is a good approximation of the activations.

$p_{\mathbf{c}_{l k}}^{\mathcal{S}}$ denotes the continuous distribution of $c_{l k}(\mathbf{x})$ over all input data $\mathbf{X}^{\mathcal{S}}=\left\{x_{1}^{\mathcal{S}}, \ldots, x_{n}^{\mathcal{S}}\right\}$, where $n$ is the number of samples in $\mathbf{X}^{\mathcal{S}}$. A second dataset, $\mathbf{X}^{\mathcal{T}}=\left\{x_{1}^{\mathcal{T}}, \ldots, x_{m}^{\mathcal{T}}\right\}$ similarly generates $p_{c l k}^{\mathcal{T}}$, where $m$ is the number of samples in $\mathbf{X}^{\mathcal{T}}$.

The representation shift $R_{l}$ is defined as the mean discrepancy $\mathcal{D}$ between the distributions over all filters $k$ in layer $l$,

$$
R_{l}\left(p^{\mathcal{S}}, p^{\mathcal{T}}\right)=\frac{1}{k} \sum_{i=1}^{k} \mathcal{D}\left(p_{\mathbf{c}_{l k}}^{\mathcal{S}}, p_{\mathbf{c}_{l k}}^{\mathcal{T}}\right),
$$

where $\mathcal{D}$ is an arbitrary discrepancy/distance metric between $p_{\mathbf{c}_{l k}}^{\mathcal{S}}$ and $p_{\mathbf{c}_{l k}}^{\mathcal{T}}$ which goes to zero when the distributions are similar. If $\mathbf{X}^{\mathcal{S}}$ and $\mathbf{X}^{\mathcal{T}}$ are statistically similar in the image domain, or if the CNN maps the datasets to similar representations, filter responses should be similar, and $R_{l}\left(p^{\mathcal{S}}, p^{\mathcal{T}}\right)$ small. We can then expect a similar classification accuracy of the datasets. If $R_{l}\left(p^{\mathcal{S}}, p^{\mathcal{T}}\right)$ is large, then filter responses has changed between the datasets, resulting in a higher risk of generalization error. The choice of layer $l$ and discrepancy metric $\mathcal{D}$ are described in Section $\mathrm{V}$ and evaluated in Section VI.

The method does not aim to do out-of-distribution or novelty detection for individual images (as e.g. [42, 41]), but aims to indicate an elevated risk of decreased classification accuracy for a dataset that is assumed to be similar to the original data. As the metric does not require annotated data, it can serve as a simple initial test to evaluate if new data (e.g. histopathology data from a new scanner) is handled well by an already trained model, i.e., if the learned feature representation applies to the new data. It can also serve as monitoring of a deployed model in a clinical environment, where changes in data preparation may introduced image changes not handled by the model, which would be detected by an increased representation shift.
Furthermore, as the metric is tightly connected to the specific model used, we expect that different models and training strategies will results in very different robustness to changes in statistics.

\section{EXPERIMENTAL SETUP AND METHODS}

In order to investigate the quantification of domain shift, we train CNNs for tumor classification and evaluate these with respect to generalization performance to unseen data. Domain generalization techniques such as color/intensity augmentation and stain normalization are evaluated, as well as a domain adaptation technique using Cycle-GANs. By evaluating how well models generalize to new target domains with and without data transformations we quantify the representation shift in each scenario, where the domain generalization and adaptation techniques are assumed to reduce both the representation shift and the drop in performance compared to a model trained without. In this section, the datasets as well as the experimental setup are described.

\section{A. Data}

The experiments were performed on two different datasets, the CAMLEYON17 dataset [52] and a dataset of colon tissue, denoted AIDA-LNCO [53].

1) CAMELYON17: This dataset consists of 500 hematoxylin and eosin (H\&E) stained lymph node whole-slide images, captured with three different scanners and collected from five medical centers [52]. The slides originating from three medical centers which were scanned by the same physical scanner (henceforth denoted Scanner 1) was chosen as training set, and data from the other scanners (Scanner 2 and Scanner 3, each originating from one of two remaining medical centers) for evaluation of the generalization performance of the trained model. As only 50 out of the 500 slides contains tumor cells, both tumor and non-tumor cells were sampled only from these 50 slides (30 of which were scanned with Scanner 1). It is possible that sampling non-tumor cells from the remaining slides would introduce some more variations, but it is not likely to affect the results to any larger extent.

The data was sampled at 0.5 microns per pixel with a patch size of $256 \times 256$ pixels. Patches where the center pixel was inside a tumor-annotated region was labelled as tumor. Nontumor patches were sampled from non-tumor tissue. 


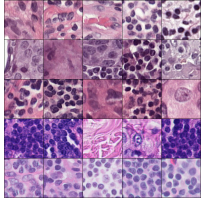

(a) Original

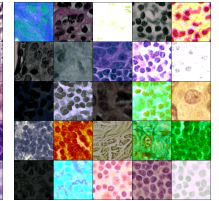

(b) Color augmentation
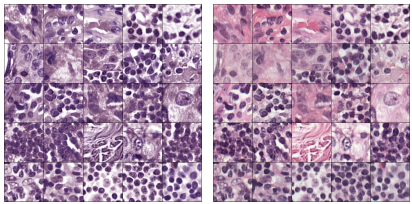

(c) Stain norm- (d) Cycle-GAN alization
Fig. 5: Five example images per center from the CAMELYON17 dataset (total 5x5 images), shown with different transformations. The three top rows per image are scanned with Scanner 1, the forth row with Scanner 2 and the last row with Scanner 3.

2) AIDA-LNCO: This dataset consists of slides of regional lymph node metastasis in colon adenocarcinoma. H\&E stained colon tissue from 89 patients from two medical centers in Sweden [53] was collected, resulting in 275 and 119 slides for each center (distributed over 27 vs 10 requests). The slides contain either primary tumor and or one or multiple lymph node sections. The data was annotated by an expert pathologist to identify tumor regions, both in primary tumor and lymph node tissue. Samples were generated from tumor and normal tissue at 0.5 microns per pixel with patch size $256 \times 256$ pixels.

\section{B. Model architecture}

Two model architectures were evaluated. One simple CNN architecture consisting of three convolutional layers (32, 64 and 128 filters per layer, kernel size $3 \times 3$ ) and one fully connected layer (512 neurons, dropout level 0.2), and a small version of GoogLeNet architecture [54] (only using the output from the first auxiliary classifier, henceforth denoted MiniGoogLeNet). We also tested larger and more high-capacity models, such as ResNet [55] and Inception-v3 [56]. However, the performance did not improve. All models were trained using the Adam optimizer (initial learning rate 0.01 , with a $10 \%$ decrease at epoch 5, 10 and 20) with geometric augmentations (random flip, rotation) and cropped at random positions to a patch size of $224 \times 224 \mathrm{px}$. All hyperparameters where selected from pre-studied cross-validation results, and were thereafter fixed throughout the experiment. The model weights were frozen after 25 training epochs. The datasets were separated at case level, making sure no patches from the same slide or patient were in both training and test sets. As test set for the CAMELYON17 dataset, 9 out of $30(30 \%)$ slides from the training set were randomly chosen, uniformly distributed over the three centers in the dataset, with the condition that the remaining training dataset had at least 10,000 samples per center and label $(\approx 40 \%$ tumor patches). For the colon dataset, 6 out of 28 requests $(\approx 20 \%$, one request contains multiple slides) were chosen randomly from the training data (roughly 100,000 patches used for training ( $\approx 60 \%$ tumor patches) and 45,000 patches for test). Each training session used the same training/test data split for all data transformation scenarios. The reported result is the average over five training sessions.

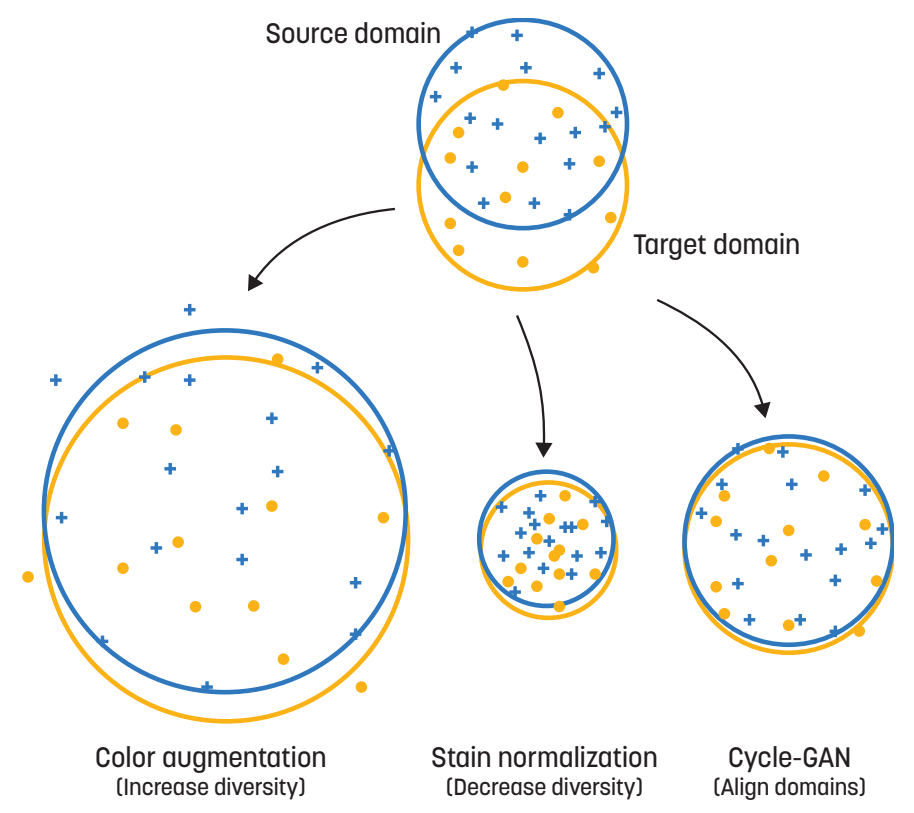

Fig. 6: Data preparation aim at increasing the intersection between domains. Different data transformation techniques do this with different strategies.

\section{Data transformations}

Three types of data transformations were included in the study (see Figure 5 for example images). Color and intensity data augmentation is a standard component in the DNN training pipeline, which can prevent over-fitting and increase generalization. Staining normalization is a specialpurpose technique that aim at normalizing differences between stained slides, by using a reference image from the source data to transform all images. Both these transformations can be seen as domain generalization strategies, since no target data is needed to define the augmentation/normalization. Style transfer using Cycle-GAN aim to transfer the "style" of one medical center to another. Style transfer is a domain adaptation technique, as sufficient data from both domains is needed in order to train the models to do the transformation.

1) Color and intensity augmentations: This transformation will henceforth be shortened color augmentation, and aim to increase image diversity in order to make the model more robust to color variations (see Figure 6). Each patch $I$ was transformed from RGB to $\mathrm{HSV}$, where each color channel $c$ was modified as $I_{c}=I_{c} \alpha+\beta$, with $\alpha$ and $\beta$ taken from uniform distributions in ranges $[1-d, 1+d]$ and $[-d, d]$ respectively. $d$ was evaluated for a range of values, and finally chosen as $d=0.5$ as this gave the best cross-dataset generalization. Choosing this value cause extreme and unnatural color variations within the dataset (see Figure 5 for example images). More moderate variations are often reported in the literature, but these were not sufficient to give a good crossdataset generalization. Tumor classification on H\&E stained images are less dependent on color information and more on pattern, making it possible to heavily augment the colors, while retaining high model accuracy. The augmentations were done online during training. 
2) Stain normalization: Commonly refers to first decomposing the image by stain colors (stain separation), and then normalizing it based on a target distribution (see Figure 6). A wide range of stain normalization techniques has been presented. For this study, the method presented in [33] was used. Each patch was normalized in relation to a reference patch, taken as a representative patch (in terms of structure and color) from one of the medical centers (scanned with Scanner 1). Since this method is computationally complex and time consuming, all patches were processed offline, before training. See Figure 5 for example images.

3) Cycle-GAN stain normalization: Cycle-GAN [24] is a method for image-to-image translation, with the goal of translating an image using a mapping $G$, from source domain to target domain (see Figure 6). In our work, we use this approach to transfer images from one medical center to another (similarly as in [22] and [23]). The goal is to learn this mapping $G$, such that the distribution of $G\left(X_{c}\right)$ is indistinguishable from $X_{1}$. To achieve this, the inverse mapping $F: X_{1} \rightarrow X_{c}$ is introduced to force $F\left(G\left(X_{c}\right)\right) \approx X_{c}$. Using this approach, features important to the target domain is transferred to $X_{c}$, and unimportant ones are unchanged. CycleGAN training uses a combination of adversarial losses and the cycle consistency loss,

$$
\begin{aligned}
\mathcal{L}_{c y c} & =\mathbb{E}_{x_{c} \sim p_{\text {data }}\left(x_{c}\right)}\left[\left\|F\left(G\left(x_{c}\right)\right)-x_{c}\right\|_{1}\right] \\
& +\mathbb{E}_{x_{1} \sim p_{\text {data }}\left(x_{1}\right)}\left[\left\|G\left(F\left(x_{1}\right)\right)-x_{1}\right\|_{1}\right],
\end{aligned}
$$

which aim to enforce a coherency between $X_{c}$ and $G\left(X_{c}\right)$. However, structural differences can be introduced in the mapping. We alleviate this problem by introducing a regularization term that confines $G\left(X_{c}\right)$ not to diverge to far from $X_{c}$ :

$$
\begin{aligned}
\mathcal{L}_{c y c}^{\prime}=\mathcal{L}_{c y c} & +\lambda \mathbb{E}_{x_{c} \sim p_{\text {data }}\left(x_{c}\right)}\left[\left\|G\left(x_{c}\right)^{*}-x_{c}^{*}\right\|\right] \\
& +\lambda \mathbb{E}_{x_{1} \sim p_{\text {data }}\left(x_{1}\right)}\left[\left\|F\left(x_{1}\right)^{*}-x_{1}^{*}\right\|\right],
\end{aligned}
$$

where $\lambda$ is used to tune the strength of the regularization $(\lambda=$ 0.05 in all of our experiments). The regularization is placed on the grayscale image, $X^{*}=\operatorname{grayscale}(X)$, which means that there is significant room for color corrections while changes in structure are confined. The regularization term is similar to the identity loss used by de Bel et al. [23]. However, in their work the loss was mainly used in order to stabilize the optimization. For examples of stain normalized images by means of the Cycle-GAN, see Figure 5.

\section{Experiments}

The domain shift analysis experiments were designed as follows. Cross-dataset generalization for tumor classification was evaluated by training $\mathrm{CNN}$ models as tumor classifiers, detecting tumor vs. non-tumor patches. The training data was separated in two sets: the first consisted of slides scanned with Scanner 1 from the CAMELYON17 dataset, the second consisted of slides from Center 1 from AIDA-LNCO. For test, both same-scanner/center data as well as unseen data from the other scanners/center was used, with results reported as patch classification accuracy, averaged over five models. Secondly, using the results from the first part, we heuristically investigate the correlation of the model accuracy with the presented metric for domain shift quantification, the representation shift, described in Section IV. Three different discrepancy metrics were evaluated: Wasserstein distance, Kullback-Leibler divergence, and Kolmogorov-Smirnov statistic.

Wasserstein distance, also known as the earth mover's distance, gives the lowest cost of "moving" one distribution to another, taking into account the amount and the distance that is needed to be moved. If $\pi\left(p_{\mathbf{c}_{l k}}^{\mathcal{S}}, p_{\mathbf{c}_{l k}}^{\mathcal{T}}\right)$ are the joined distributions with margins $p_{\mathbf{c}_{l k}}^{\mathcal{S}}$ and $p_{\mathbf{c}_{l k}}$, the Wasserstein distance between the distributions is given by:

$$
W\left(p_{\mathbf{c}_{l k}}^{\mathcal{S}}, p_{\mathbf{c}_{l k}}^{\mathcal{T}}\right)=\inf _{\pi \in \Gamma\left(p_{\mathbf{c}_{l k}}^{\mathcal{S}}, p_{\mathbf{c}_{l k}}^{\mathcal{T}}\right)} \int_{R x R}|(x-y)| d \pi(x, y) .
$$

The distance depends on the absolute values of the generated distributions, and will thus not only measure the shape discrepancy but also the distance between two distributions. In this setting, the scale of the metric will depend on the model architecture and the dataset.

Kullback-Leibler (KL) divergence is defined as

$$
K L(P \| Q)=\sum_{x \in X} P(x) \log \left(\frac{P(x)}{Q(x)}\right),
$$

where $P$ and $Q$ are discrete probability distributions. Here, we define it as the divergence from the target domain to the source domain, where the discrete probability density functions are approximated from the samples with 100 values (range determined by the minimum and maximum values of the samples of the two distributions which are compared). A small constant $\left(10^{-3}\right)$ was added where the discrete distributions are zero, for numerical stability.

Kolmogorov-Smirnov (KS) statistic [57] is calculated by estimating the cumulative distribution functions using the empirical samples from two random variables (in this case activations from two different datasets), then taking the maximum absolute distance between them, bounded between 0 and 1. It measures the shape difference between distributions, but does not take into account the distance separating them.

For the Simple model, the representation shifts for all three convolutional layers were calculated. For Mini-GoogleNet, four layers were chosen. The Pearson correlation [58] between the accuracy and representation shift for each layer was calculated per training sessions, with the mean correlation presented per metric, layer, model and dataset.

\section{E. Evaluation}

The domain generalization results are evaluated by comparing patch level accuracy when testing on source vs target datasets. To demonstrate the ability of model specific domain shift detection, we compare our proposed representation shift with two existing methods. The first method, A-distance [59], was introduced in domain adaptation theory as a way of measuring differences between source and target datasets. It can be approximated by training a linear SVM to discriminate between source and target activations, with $d_{A}=2(1-2 \epsilon)$, where $\epsilon$ is the lowest error of the classifier. A-distance is a linear metric, were 0 indicate total overlap of data representations (no domain shift) and 2 indicate fully separated 


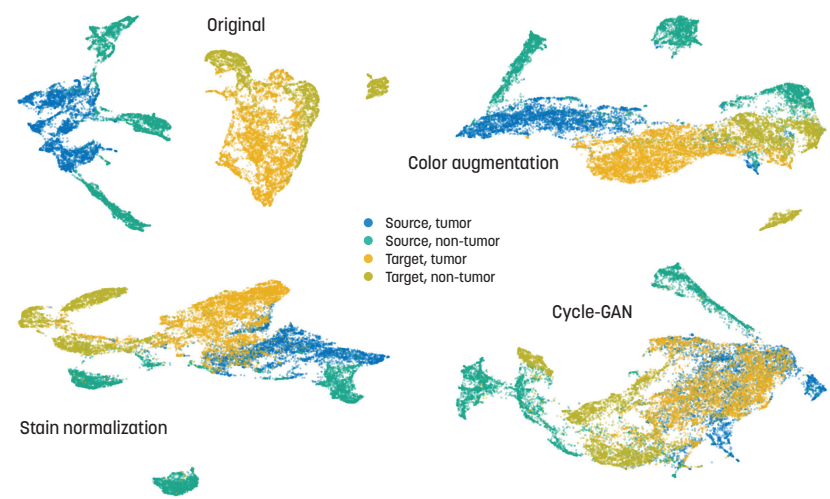

Fig. 7: UMAP embeddings [61] of the mean activations $c_{l k}$ for $10 \mathrm{~K}$ images from the training dataset (source) and $10 \mathrm{~K}$ images from the unseen dataset (target), where source and target are Scanner 1 and Scanner 3 of the CAMELYON17 dataset, respectively. $c_{l k}$ are taken from the last convolutional layer of the Simple CNN. The four different plots show how the data transformations impact the feature representation embedding. With training on original data, the domains are completely disjoint, while the transformations can more or less successfully align the domains in the representation space. The visualizations correspond to the representation space illustration rightmost in Figure 1.

representations. We use the same activations for calculating the A-distance as we use for calculation of the representation shift. The second method comes from the deep uncertainty community. Ovadia et. al. [60] compared different methods for quantifying predictive uncertainty under dataset shifts, and concluded that ensembles of $N$ networks resulted in the best calibrated measure. We therefore use Deep Ensambles [49] with $N=5$ to estimate the risk of performance drop. The same training/test-data split was repeated five time, resulting in five ensembles à five models, for each dataset, model architecture and transformation (total of 400 trained models). For each ensemble, the mean probability for each test sample was taken over the (five) models' softmax outputs. The uncertainty over the entire dataset is calculated as $1-\hat{p}$, where $\hat{p}$ is an estimation of the true (supervised) accuracy $p$, and is calculated as the mean probability over all test samples. For a well-calibrated model $1-\hat{p}$ maps to $1-p$. The reported accuracy and uncertainty is the average over all (five) ensembles.

\section{RESUlTS}

We first present results on performance and changes in latent representation across domain shifts, followed by results of the proposed representation shift measure.

\section{A. Cross-dataset Generalization}

Table I shows validation accuracies when training a model on data from one domain, and evaluating it on another. Both model architectures and datasets suffer from poor generalization when no augmentation is used, with considerable drop in mean accuracy. The three data transformations generally resulted in higher performance on the target domain(s), and for the CAMELYON17 data also on the source domain (Scanner 1). Data transformation with Cycle-GAN gave higher performing models on CAMELYON17, but was not as successful on AIDA-LNCO. The results are not conclusive in terms of which method to prefer, but show that some domain generalization/adaptation is needed for robust performance. Figure 7 shows an example of a visual interpretation of the representation space of the last convolutional layer in the Simple CNN. The embeddings have been performed by means of the UMAP dimensionality reduction method [61] on the mean filter activations $c_{l k}$ in Equation 1. The source dataset is Scanner 1 of the CAMELYON17 dataset, while the target is Scanner 3 data. Inspecting the embedding when no transformations was used for training and inference, the two datasets are completely disjoint, indicating high probability of generalization problems for the classifier to the unseen domain. The different data transformation strategies, on the other hand, can to some extent align the target domain with the source domain. Best alignment seems to be generated with the Cycle-GAN transformation, where the tumor classes of the two datasets almost have full intersection. This also agrees with the generalization performance of Simple CNN to Scanner 3 in Table I. It is also interesting with a consistent clustering into 3 separate regions for the non-tumor class of the source domain. One possible explanation could be that the data is composed of images from 3 different medical centers, but scanned with the same physical scanner, and that this is mostly affecting the representation of the non-tumor images.

\section{B. Representation shift}

Table II shows the Pearson correlation between the representation shift (Equation 2) and the model accuracy, per layer, dataset and model architecture. This metric is limited to only give the linear correlation, while it may well not be strictly linear, especially considering that the model accuracy depends on the final fully connected layers. However, as seen in the table, there is a strong negative correlation, which varies somewhat depending on layer, model and dataset, and with the discrepancy metric used. The Wasserstein distance $\left(R_{W}\right)$ gives overall high correlation values, possibly explained by its intrinsic property of comparing both shape and position of the distributions. Comparative plots for domain shift metrics are shown for CAMLEYON17 dataset in Figure 8, and AIDALNCO in Figure 9. The left-most column shows the representation shift using the Wasserstein distance, $R_{W}$, for layer 3 of the Simple model and layer 1 of the Mini-GoogLeNet, in relation to model patch level accuracy for all models trained (5 per experimental setup). The other two columns show the corresponding results for A-distance and Deep Ensembles. The blue line shows the linear regression line (shaded blue area gives the 95\% confidence interval), which is added for visualization of the correlation. Models trained with original data (shown with circular markers) show significant drop in accuracy when evaluated on target data. This correlates well with higher values for the representation shift and A-distance, while only slight increase in uncertainty for Deep Ensembles. As the drop in accuracy is reduced with all data transformation 
TABLE I: Class balanced mean accuracies (standard deviation) (\%), training a tumor classifier on Scanner 1 for CAMELYON17, and Center 1 for AIDA-LNCO, testing it on same-scanner/center data and other data, using two different model architectures, evaluated across 5 independent training runs.

CAMELYON17

\begin{tabular}{|c|c|c|c|c|c|c|c|c|}
\hline & Simple CNN & & & & Mini-Googl & & & \\
\hline Train & Scanner 1 & Scanner 2 & Scanner 3 & $\begin{array}{l}\text { Mean drop } \\
\text { (p.p.) }\end{array}$ & Scanner 1 & Scanner 2 & Scanner 3 & $\begin{array}{l}\text { Mean drop } \\
\text { (p.p.) }\end{array}$ \\
\hline Orig. data & $90.0(5.0)$ & $48.9(1.2)$ & $72.3(12.4)$ & $29.5(9.7)$ & $95.4(2.5)$ & $49.7(3.4)$ & $73.8(12.8)$ & $33.6(7.4)$ \\
\hline Color aug. & $94.5(1.3)$ & $91.4(0.8)$ & $89.3(3.9)$ & $4.1(1.7)$ & $96.4(0.7)$ & $90.3(0.2)$ & $93.5(1.1)$ & $4.5(0.5)$ \\
\hline Stain norm. & $94.1(2.1)$ & $93.5(1.3)$ & $92.7(2.0)$ & $0.9(1.5)$ & $97.8(1.0)$ & $93.5(2.5)$ & $96.5(0.7)$ & $2.8(1.3)$ \\
\hline Cycle-GAN & $95.0(1.3)$ & $91.0(1.7)$ & $91.8(0.6)$ & $3.6(0.8)$ & $97.0(0.7)$ & $90.1(2.5)$ & $94.4(0.4)$ & $4.7(1.6)$ \\
\hline
\end{tabular}

AIDA-LNCO

\begin{tabular}{l||c|c||c||c|c||c}
\multicolumn{1}{c|}{ Test } & $\begin{array}{c}\text { Simple CNN } \\
\text { Center 1 }\end{array}$ & Center 2 & $\begin{array}{c}\text { Mean drop } \\
\text { (p.p.) }\end{array}$ & Center 1 & Center 2 & $\begin{array}{c}\text { Mean drop } \\
\text { (p.p.) }\end{array}$ \\
\hline Train & $98.3(0.8)$ & $73.3(6.6)$ & $25.0(5.8)$ & $97.3(1.3)$ & $72.8(8.1)$ & $24.5(7.5)$ \\
Orig. data & $96.0(3.3)$ & $95.8(1.0)$ & $0.3(2.5)$ & $95.3(4.6)$ & $95.5(2.0)$ & $-0.2(2.7)$ \\
Color aug. & $96.3(4.0)$ & $95.5(3.1)$ & $0.8(0.9)$ & $95.4(3.7)$ & $95.7(2.6)$ & $-0.3(2.0)$ \\
Stain norm. & $98.3(1.2)$ & $77.7(3.4)$ & $20.6(3.6)$ & $97.2(1.3)$ & $81.7(10.7)$ & $15.5(10.5)$ \\
Cycle-GAN & & &
\end{tabular}

TABLE II: Pearson correlations between different metrics for the representation shift $R$ as a function of layer $l . R_{W}, R_{K L}$ and $R_{K S}$ denote calculating $R$ using the Wasserstein distance, the KL-divergence or the KS-statistic. Layer 3 and Layer 4 are the last convolution layers in the Simple and Mini-GoogleNet architectures respectively. Bold values denote the largest negative correlation per model and dataset.

\begin{tabular}{l||c|c|c||c|c|c|c}
\multicolumn{1}{l||}{} & \multicolumn{2}{l}{ Simple CNN } \\
\multicolumn{1}{l|}{ CAMELYON17 } & Layer 1 & Layer 2 & Layer 3 & Layer 1 & Layer 2 & Layer 3 & Layer 4 \\
\hline$R_{W}$ & -0.941 & -0.951 & $-\mathbf{0 . 9 6 5}$ & $-\mathbf{0 . 9 2 6}$ & -0.860 & -0.856 & -0.891 \\
$R_{K L}$ & -0.789 & -0.825 & -0.865 & -0.665 & -0.639 & -0.736 & -0.833 \\
$R_{K S}$ & -0.756 & -0.827 & -0.848 & -0.701 & -0.679 & -0.771 & -0.833 \\
\hline AIDA-LNCO & -0.941 & -0.927 & $-\mathbf{0 . 9 4 4}$ & -0.889 & $-\mathbf{0 . 9 0 0}$ & -0.848 & -0.763 \\
\hline$R_{W}$ & -0.893 & -0.909 & -0.919 & -0.883 & -0.885 & -0.871 & -0.832 \\
$R_{K L}$ & -0.850 & -0.849 & -0.877 & -0.753 & -0.770 & -0.787 & -0.828 \\
$R_{K S}$
\end{tabular}

techniques, so is the representation shift, for both models and datasets. A-distance handles this to some extend, but especially for CAMLEYON17 it remains very high even as the performance drop is almost zero. Deep Ensembles is in many cases unable to detect the dataset shift for original data, and retains a low uncertainty, even as the true accuracy drops significantly. It seems to give better calibrated uncertainty when the dataset shift is lower, i.e., when data transformation is used. However, for AIDA-LNCO, the uncertainty remains almost constant for Mini-GoogLeNet, and while the Simple $\mathrm{CNN}$ gets a slightly more pronounced negative correlation, the drop is very low (approx. 8 p.p.), while the true performance drops much more (approx. 20 p.p.).

Table III shows the Pearson correlation between the accuracy and the three metrics, showing overall higher correlation between representation shift and accuracy as compared to the other methods.

\section{DISCUSSION}

The results shown in Table I are consistent with previous literature demonstrating that some kind of domain generalization/adaption is crucial for creating robust models which
TABLE III: Pearson correlations between accuracy and metrics for measuring domain shift. Bold values indicate the largest negative correlation per model and dataset.

\begin{tabular}{l||c|c|c} 
CAMELYON17 & Representation shift & A-distance [59] & Deep Ensembles [49] \\
\hline Simple CNN & $\mathbf{- 0 . 9 6 5}$ & -0.435 & -0.177 \\
Mini-GoogLeNet & $\mathbf{- 0 . 9 2 6}$ & -0.488 & -0.573 \\
\hline AIDA-LNCO & $\mathbf{- 0 . 9 4 4}$ & -0.896 & -0.918 \\
\hline Simple CNN & $\mathbf{- 0 . 8 8 9}$ & -0.833 & -0.328 \\
Mini-GoogLeNet &
\end{tabular}

can handle data variations that may occur in clinical practice. Together with Figure 7, this indeed confirms the picture illustrated in Figure 1, that a small difference in image characteristics can result in completely separated distributions in the representation domain. The graphs in Figure 8 and Figure 9, and correlation coefficients in Table II, show that this can be measured with the representation shift metric, as scenarios where source and target data are separated give a high drop in accuracy and a large representation shift.

From Table II we see an overall high correlation between 


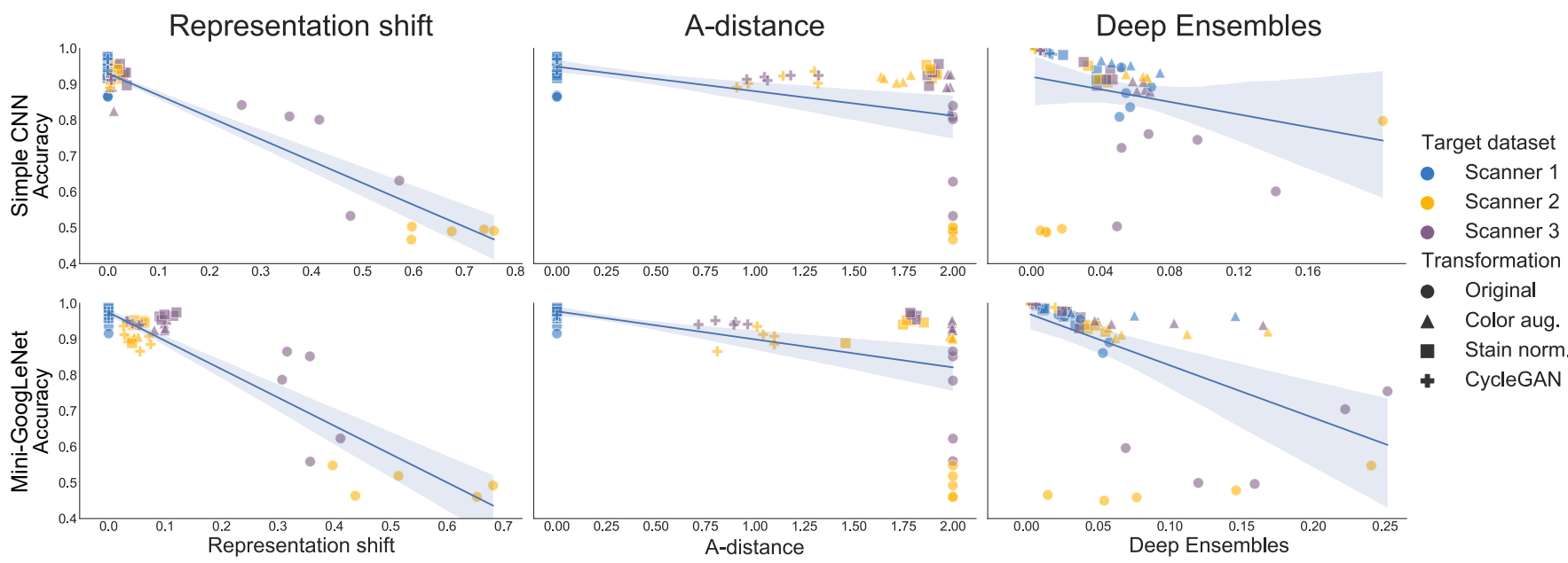

Fig. 8: Dataset: CAMELYON17. Relation between patch level accuracy (see Table I) and representation shift $R_{W}$ (Eq. 2), A-distance [59], and Deep Ensembles [49], with and without data transformation. Representation shift and A-distance are calculated from layer 3 in the Simple CNN, and from layer 1 in the Mini-GoogLeNet. A regression line is added to show the correlation between the variables, with the shaded blue area denoting the $95 \%$ confidence interval. Please note different $\mathrm{x}$-axis scales. Best viewed digitally.

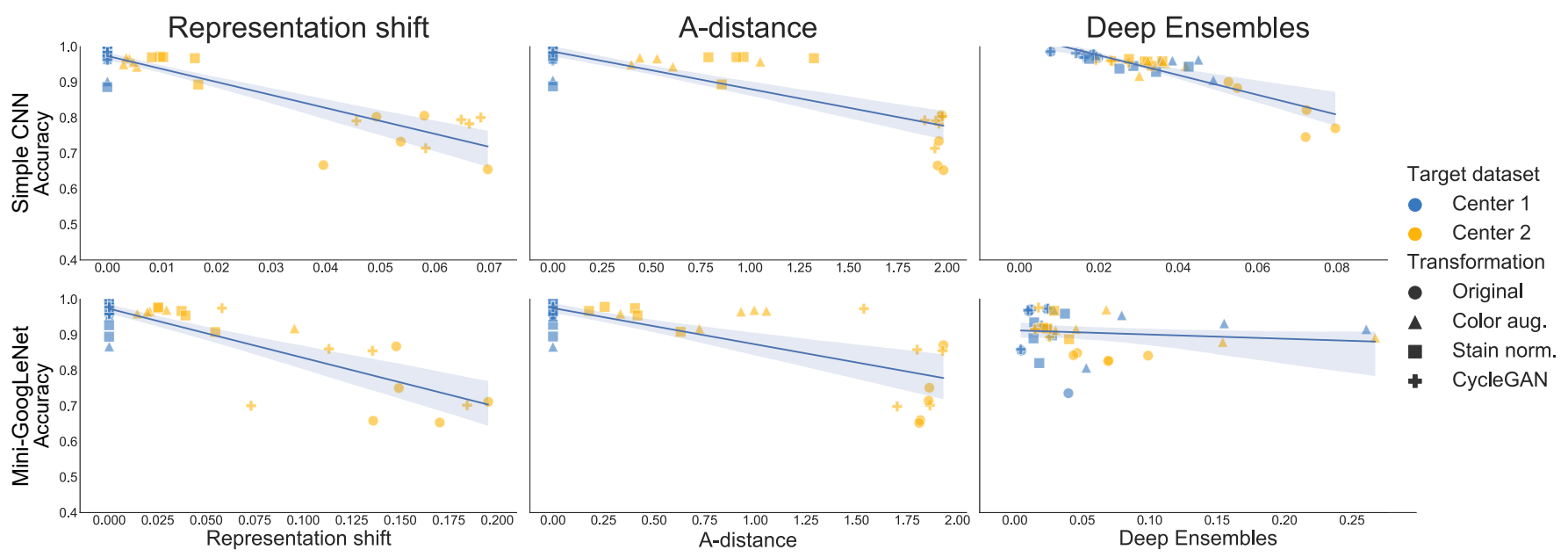

Fig. 9: Dataset: AIDA-LNCO. See Figure 8 for details.

the representation shift and model accuracy for both early and deep layers. Large differences in early layers are likely to propagate through the model. In the case of Mini-GoogLeNet, this phenomena is less pronounced. One hypothesis for this is that many of the filter responses of previous layers are not used by deeper layers.

Comparing representation shift with other metrics demonstrates its ability to provide a robust estimation of performance drop with increased domain shift, over a wide range of domain shifts. However, as it is an unsupervised metric, it is classagnostic. This means that it is not possible to fully detect errors which occur when distributions are overlapping but samples are of different classes. This shortage is shared with A-distance. Additionally, the representation shift measure is not bounded to a fixed interval, making it hard to compare absolute values between models and datasets. For a specific model, however, it is easy to calibrate the representation shift vs accuracy, in order to get robust indications of risk of performance drop due to domain shift in unseen data.

Before a CNN can be used in a clinical setting, it needs to be properly validated. This validation should include evaluation on data with more variance than included in the training set, to affirm model performance. However, it is not possible to cover all data variations that may occur. Using the representation shift it is possible to do both an initial control on a large amount of unlabelled data, and continuous monitoring of a deployed model. By monitoring a model's data representation, differences in data statistics and model performance degradation can be detected.

In future work, we will further investigate how the representation shift measure can be refined, how dependent it is on the number of data samples, and how it can be used as an objective for improved domain generalization or adaptation. A similar formulation can possibly also be used for out-of-distribution 
detection. Furthermore, interesting future work regards how representation shift can be used for domain adaptation between tissue types or modalities, as well as evaluation of other domain adaptation techniques.

\section{CONCLUSION}

Detecting domain shift is important in order for deep learning models to be used for histopathology applications in clinical practice, as differences in distribution of new data may lead to decreased model performance and in worst case patient risk. In this paper we highlighted how small statistical variations in the input data can result in large difference in the learned representation of convolutional neural networks. We presented a measure, the representation shift, enabling us to quantify this model specific domain shift in the representation domain, and we demonstrated how it correlates with increased risk of performance degradation. The measure can therefore be used to analyze model performance on novel unlabelled datasets, as well as monitoring clinically deployed models over time. This is one step towards safe deployment of deep convolutional models for clinical applications in histopathology.

\section{ACKNOWLEDGMENT}

This work was supported by the Wallenberg AI and Autonomous Systems and Software Program (WASP-AI), the research environment ELLIIT, and AIDA Vinnova grant 2017 02447.

\section{REFERENCES}

[1] J. Quionero-Candela, M. Sugiyama, A. Schwaighofer, and N. D. Lawrence, Dataset Shift in Machine Learning. The MIT Press, 2009.

[2] A. Torralba and A. A. Efros, "Unbiased look at dataset bias," in $C V P R$ 2011, 2011.

[3] A. Kurakin, I. Goodfellow, and S. Bengio, "Adversarial examples in the physical world," ICLR Workshop, 2017.

[4] W. M. Kouw and M. Loog, "A review of domain adaptation without target labels," IEEE TPAMI, 2019.

[5] Y. Yagi, "Color standardization and optimization in Whole Slide Imaging," BioMed Central, Report, 2011.

[6] K. Stacke, G. Eilertsen, J. Unger, and C. Lundström, "A Closer Look at Domain Shift for Deep Learning in Histopathology," arXiv:1909.11575 [cs], 2019.

[7] C. Szegedy, W. Zaremba, I. Sutskever, J. Bruna, D. Erhan, I. Goodfellow, and R. Fergus, "Intriguing properties of neural networks," arXiv:1312.6199 [cs], 2014.

[8] I. Goodfellow, J. Pouget-Abadie, M. Mirza, B. Xu, D. WardeFarley, S. Ozair, A. Courville, and Y. Bengio, "Generative Adversarial Nets," in Advances in Neural Information Processing Systems 27, 2014.

[9] A. Azulay and Y. Weiss, "Why do deep convolutional networks generalize so poorly to small image transformations?" arXiv:1805.12177 [cs], 2019.

[10] L. Engstrom, B. Tran, D. Tsipras, L. Schmidt, and A. Madry, "Exploring the Landscape of Spatial Robustness," in International Conference on Machine Learning, 2019, pp. 1802-1811.

[11] S. Zheng, Y. Song, T. Leung, and I. Goodfellow, "Improving the Robustness of Deep Neural Networks via Stability Training," 2016.

[12] E. Tzeng, J. Hoffman, N. Zhang, K. Saenko, and T. Darrell, "Deep Domain Confusion: Maximizing for Domain Invariance," arXiv:1412.3474 [cs], 2014.
[13] M. Long, Y. Cao, J. Wang, and M. I. Jordan, "Learning Transferable Features with Deep Adaptation Networks," in Proceedings of the 32nd International Conference on Machine Learning, vol. W\&CP volume 37. JMLR, 2015, p. 9.

[14] M. Long, H. Zhu, J. Wang, and M. I. Jordan, "Deep Transfer Learning with Joint Adaptation Networks," in Proceedings of the 34th International Conference on Machine Learning Volume 70, ser. ICML'17. JMLR, 2017, pp. 2208-2217.

[15] H. Ajakan, P. Germain, H. Larochelle, F. Laviolette, and M. Marchand, "Domain-Adversarial Neural Networks," arXiv:1412.4446 [cs, stat], 2015.

[16] Y. Ganin, E. Ustinova, H. Ajakan, P. Germain, H. Larochelle, F. Laviolette, M. Marchand, and V. Lempitsky, "Domainadversarial Training of Neural Networks," J. Mach. Learn. Res., vol. 17, no. 1, pp. 2096-2030, 2016.

[17] E. Tzeng, J. Hoffman, K. Saenko, and T. Darrell, "Adversarial Discriminative Domain Adaptation," in Proceedings of the IEEE Conference onComputer Vision and Pattern Recognition, 2017, pp. 2962-2971.

[18] M. Wang and W. Deng, "Deep visual domain adaptation: A survey," Neurocomputing, vol. 312, pp. 135-153, 2018.

[19] M. W. Lafarge, J. P. W. Pluim, K. A. J. Eppenhof, P. Moeskops, and M. Veta, "Domain-Adversarial Neural Networks to Address the Appearance Variability of Histopathology Images," in Deep Learning in Medical Image Analysis and Multimodal Learning for Clinical Decision Support, ser. Lecture Notes in Computer Science. Cham: Springer International Publishing, 2017, pp. 83-91.

[20] F. G. Zanjani, S. Zinger, B. E. Bejnordi, J. A. W. M. van der Laak, and P. H. N. de With, "Stain normalization of histopathology images using generative adversarial networks," in 2018 IEEE 15th International Symposium on Biomedical Imaging (ISBI 2018), 2018.

[21] J. Ren, I. Hacihaliloglu, E. A. Singer, D. J. Foran, and X. Qi, "Unsupervised Domain Adaptation for Classification of Histopathology Whole-Slide Images," Frontiers in Bioengineering and Biotechnology, vol. 7, May 2019. [Online]. Available: https://www.ncbi.nlm.nih.gov/pmc/articles/PMC6529804/

[22] M. T. Shaban, C. Baur, N. Navab, and S. Albarqouni, "StainGAN: Stain Style Transfer for Digital Histological Images," in arXiv:1804.01601 [cs], 2019.

[23] T. d. Bel, M. Hermsen, J. Kers, J. v. d. Laak, and G. Litjens, "Stain-Transforming Cycle-Consistent Generative Adversarial Networks for Improved Segmentation of Renal Histopathology," in International Conference on Medical Imaging with Deep Learning, 2019, pp. 151-163.

[24] J.-Y. Zhu, T. Park, P. Isola, and A. A. Efros, "Unpaired Imageto-Image Translation Using Cycle-Consistent Adversarial Networks," in 2017 IEEE International Conference on Computer Vision (ICCV), 2017, pp. 2242-2251.

[25] F. Xing, T. Bennett, and D. Ghosh, "Adversarial Domain Adaptation and Pseudo-Labeling for Cross-Modality Microscopy Image Quantification," in Medical Image Computing and Computer Assisted Intervention - MICCAI 2019, ser. Lecture Notes in Computer Science. Cham: Springer International Publishing, 2019, pp. 740-749.

[26] K. Muandet, D. Balduzzi, and B. Schölkopf, "Domain Generalization via Invariant Feature Representation," in Proceedings of the 30th International Conference on International Conference on Machine Learning - Volume 28, ser. ICML'13. JMLR.org, 2013, pp. I-10-I-18.

[27] M. Ghifary, W. B. Kleijn, M. Zhang, and D. Balduzzi, "Domain Generalization for Object Recognition with Multi-task Autoencoders," in 2015 IEEE International Conference on Computer Vision (ICCV). Santiago, Chile: IEEE, 2015.

[28] H. Li, S. J. Pan, S. Wang, and A. C. Kot, "Domain Generalization with Adversarial Feature Learning," in 2018 IEEE/CVF Conference on Computer Vision and Pattern Recognition, 2018, pp. 5400-5409. 
[29] R. Volpi, H. Namkoong, O. Sener, J. Duchi, V. Murino, and S. Savarese, "Generalizing to Unseen Domains via Adversarial Data Augmentation," in Proceedings of the 32Nd International Conference on Neural Information Processing Systems, ser. NIPS'18. USA: Curran Associates Inc., 2018, pp. 5339-5349.

[30] J. Tobin, R. Fong, A. Ray, J. Schneider, W. Zaremba, and P. Abbeel, "Domain randomization for transferring deep neural networks from simulation to the real world," in 2017 IEEE/RSJ International Conference on Intelligent Robots and Systems (IROS), 2017, pp. 23-30.

[31] J. Tremblay, A. Prakash, D. Acuna, M. Brophy, V. Jampani, C. Anil, T. To, E. Cameracci, S. Boochoon, and S. Birchfield, "Training Deep Networks with Synthetic Data: Bridging the Reality Gap by Domain Randomization," in 2018 IEEE/CVF Conference on Computer Vision and Pattern Recognition Workshops (CVPRW), 2018, pp. 1082-10828.

[32] D. Tellez, M. Balkenhol, N. Karssemeijer, G. Litjens, J. v. d. Laak, and F. Ciompi, "H and $\mathrm{E}$ stain augmentation improves generalization of convolutional networks for histopathological mitosis detection," in Medical Imaging 2018: Digital Pathology, vol. 10581. International Society for Optics and Photonics, 2018, p. $105810 Z$.

[33] A. Vahadane, T. Peng, A. Sethi, S. Albarqouni, L. Wang, M. Baust, K. Steiger, A. M. Schlitter, I. Esposito, and N. Navab, "Structure-Preserving Color Normalization and Sparse Stain Separation for Histological Images," IEEE Transactions on Medical Imaging, vol. 35, no. 8, pp. 1962-1971, 2016.

[34] A. Bentaieb and G. Hamarneh, "Adversarial Stain Transfer for Histopathology Image Analysis," IEEE Transactions on Medical Imaging, vol. 37, no. 3, pp. 792-802, 2018.

[35] I. Arvidsson, N. C. Overgaard, F.-E. Marginean, A. Krzyzanowska, A. Bjartell, K. Åström, and A. Heyden, "Generalization of prostate cancer classification for multiple sites using deep learning," in 2018 IEEE 15th International Symposium on Biomedical Imaging (ISBI 2018), 2018, pp. 191-194.

[36] F. Ciompi, O. Geessink, B. E. Bejnordi, G. S. de Souza, A. Baidoshvili, G. Litjens, B. van Ginneken, I. Nagtegaal, and J. van der Laak, "The importance of stain normalization in colorectal tissue classification with convolutional networks," in 2017 IEEE 14th International Symposium on Biomedical Imaging (ISBI 2017), 2017, pp. 160-163.

[37] D. Tellez, G. Litjens, P. Bandi, W. Bulten, J.-M. Bokhorst, F. Ciompi, and J. van der Laak, "Quantifying the effects of data augmentation and stain color normalization in convolutional neural networks for computational pathology," arXiv:1902.06543 [cs], 2019.

[38] R. Geirhos, P. Rubisch, C. Michaelis, M. Bethge, F. A. Wichmann, and W. Brendel, "ImageNet-trained CNNs are biased towards texture; increasing shape bias improves accuracy and robustness," arXiv:1811.12231 [cs, q-bio, stat], 2018.

[39] N. Frosst, N. Papernot, and G. Hinton, "Analyzing and Improving Representations with the Soft Nearest Neighbor Loss," arXiv:1902.01889 [cs, stat], 2019.

[40] N. S. Keskar, D. Mudigere, J. Nocedal, M. Smelyanskiy, and P. T. P. Tang, "On Large-Batch Training for Deep Learning: Generalization Gap and Sharp Minima," ArXiv, vol. abs/1609.04836, 2016.

[41] A. Schultheiss, C. Käding, A. Freytag, and J. Denzler, "Finding the Unknown: Novelty Detection with Extreme Value Signatures of Deep Neural Activations," in Pattern Recognition, ser. Lecture Notes in Computer Science. Cham: Springer International Publishing, 2017, pp. 226-238.

[42] N. Papernot and P. McDaniel, "Deep k-Nearest Neighbors: Towards Confident, Interpretable and Robust Deep Learning," arXiv:1803.04765 [cs, stat], 2018.

[43] M. Raghu, C. Zhang, J. M. Kleinberg, and S. Bengio, "Transfusion: Understanding Transfer Learning with Applications to Medical Imaging,” ArXiv, vol. abs/1902.07208, 2019.
[44] D. Erhan, Y. Bengio, A. Courville, and P. Vincent, "Visualizing Higher-Layer Features of a Deep Network," Technical Report, Univeristé de Montréal, 2009.

[45] C. Olah, A. Mordvintsev, and L. Schubert, "Feature Visualization," Distill, vol. 2, no. 11, p. e7, 2017.

[46] J. Deng, W. Dong, R. Socher, L. Li, Kai Li, and Li Fei-Fei, "ImageNet: A large-scale hierarchical image database," in 2009 IEEE Conference on Computer Vision and Pattern Recognition, 2009, pp. 248-255.

[47] Y. Gal, "Uncertainty in Deep Learning," Ph.D. dissertation, University of Cambridge, 2016.

[48] C. Guo, G. Pleiss, Y. Sun, and K. Q. Weinberger, "On Calibration of Modern Neural Networks," in Proceedings of the 34th International Conference on Machine Learning, vol. 70. PMLR, 2017, p. 10.

[49] B. Lakshminarayanan, A. Pritzel, and C. Blundell, "Simple and Scalable Predictive Uncertainty Estimation using Deep Ensembles," in Advances in Neural Information Processing Systems 30. Curran Associates, Inc., 2017, pp. 6402-6413.

[50] J. Yosinski, J. Clune, Y. Bengio, and H. Lipson, "How transferable are features in deep neural networks?" in Advances in Neural Information Processing Systems 27. Curran Associates, Inc., 2014, pp. 3320-3328.

[51] B. Sun and K. Saenko, "Deep CORAL: Correlation Alignment for Deep Domain Adaptation," in Computer Vision - ECCV 2016 Workshops. Springer, Cham, 2016, pp. 443-450.

[52] G. Litjens, P. Bandi, B. Ehteshami Bejnordi, O. Geessink, M. Balkenhol, P. Bult, A. Halilovic, M. Hermsen, R. van de Loo, R. Vogels, Q. F. Manson, N. Stathonikos, A. Baidoshvili, P. van Diest, C. Wauters, M. van Dijk, and J. van der Laak, "1399 H\&E-stained sentinel lymph node sections of breast cancer patients: the CAMELYON dataset," Gigascience, vol. 7, no. 6, 2018.

[53] G. Maras, M. Lindvall, and C. Lundstrom, "Regional lymph node metastasis in colon adenocarcinoma," 2019. [Online]. Available: doi:10.23698/aida/lnco

[54] C. Szegedy, W. Liu, Y. Jia, P. Sermanet, S. Reed, D. Anguelov, D. Erhan, V. Vanhoucke, and A. Rabinovich, "Going deeper with convolutions," in 2015 IEEE Conference on Computer Vision and Pattern Recognition (CVPR), 2015, pp. 1-9.

[55] K. He, X. Zhang, S. Ren, and J. Sun, "Deep Residual Learning for Image Recognition," in 2016 IEEE Conference on Computer Vision and Pattern Recognition (CVPR), 2016, pp. 770-778.

[56] C. Szegedy, V. Vanhoucke, S. Ioffe, J. Shlens, and Z. Wojna, "Rethinking the Inception Architecture for Computer Vision," 2016 IEEE Conference on Computer Vision and Pattern Recognition (CVPR), pp. 2818-2826, 2015.

[57] "Kolmogorov-Smirnov Test," in The Concise Encyclopedia of Statistics. New York, NY: Springer New York, 2008, pp. 283287.

[58] "Pearson's Correlation Coefficient," in Encyclopedia of Public Health. Dordrecht: Springer Netherlands, 2008, pp. 10901091

[59] S. Ben-David, J. Blitzer, K. Crammer, and F. Pereira, "Analysis of Representations for Domain Adaptation," in Advances in Neural Information Processing Systems 19. MIT Press, 2007, pp. 137-144.

[60] Y. Ovadia, J. Snoek, E. Fertig, B. Lakshminarayanan, S. Nowozin, D. Sculley, J. Dillon, J. Ren, and Z. Nado, "Can you trust your model's uncertainty? Evaluating predictive uncertainty under dataset shift," in Advances in Neural Information Processing Systems 32. Curran Associates, Inc., 2019, pp. 13 991-14002.

[61] L. McInnes, J. Healy, and J. Melville, "Umap: Uniform manifold approximation and projection for dimension reduction," arXiv preprint arXiv:1802.03426, 2018. 